\title{
Evaluation of the Fundamental Physical Constants in Mathematica
}

\author{
Andrey S. Siver \\ Institute of High Energy Physics, Protvino, Russia
}

\begin{abstract}
We present PAREVAL package consisting of a number of Mathematica modules used to re-evaluate basic CODATA:1998 recommended fundamental physical constants (FPC). Package can be found at http://sirius.ihep.su/ siver. Values of the basic FPC-1998 with the positive defined correlation matrix and a number of functions for the FPC usage can be found therein. Among other PAREVAL functions there are functions for energy levels of hydrogen and deuterium, electron and muon magnetic moment anomaly and muonium ground-state hyperfine splitting.
\end{abstract}

\section{Introduction}

Hopes for the discovery of new physics at present time are frequently connected with high-precision experiments combined with corresponding high-precision calculations. These calculations usually involve values of the FPC and should also use their uncertainties and correlation matrix. But none of the available resources provides the last one (correlation coefficients presented on the official site [3] are incorrect because they are rounded to three decimal figures).

Design of PAREVAL package has been mostly motivated by two facts:

- Methodological doubt about validity of CODATA recommended FPC-1998 [1. This doubt arises due to the non-positive semi definiteness of the correlation matrix of the input experimental data presented in 11. More information can be found in [4]

- Absence of powerful IT resources for scientific activities in FPC studies. Critical notes about several resources can be found in [4]

Our FPC-1998 re-evaluation has been generally based on review [1]. We have checked the values of the basic FPC and got their correlation matrix. The rest (derived) FPC can be expressed as a functions of the basic ones and thus be calculated.

\section{Package Structure}

PAREVAL consists of a number of Mathematica [2] modules which can be ranged as followings: 
1. Modules for FPC usage. Contains data and functions for the FPC usage in high-precision calculations;

2. Modules with physical formulae collection. Contains functions designed to calculate energy levels of hydrogen and deuterium, electron and muon magnetic moment anomaly, muonium ground-state hyperfine splitting and some other functions;

3. Modules for experimental data processing. Contains functions transforming experimental data between input and internal forms;

4. Module for parameters evaluation. Contains Mathematica functions for evaluation of parameters based on least-squares method;

5. Modules for results presentation. Contains functions used to present results of parameters evaluation in $\mathrm{LT}_{\mathrm{E} X} \mathrm{X}$ system.

The package has general public license (GPL) [5] and can be found at http://sirius.ihep.su/ siver. Values of the basic FPC-1998 with the positive defined correlation matrix, a number of useful functions for the FPC usage, Mathematica notebook in which calculations have been carried out can be found therein.

Most important modules are in a nutshell described below.

\subsection{Modules for FPC Usage}

The first module contains several Mathematica variables which can be used in calculations. The second one contains a function for the calculation of the FPC uncertainties propagation with FPC covariance matrix.

\begin{tabular}{|c|c|c|c|c|c|}
\hline Module & Function & Input & Output & Example & Description of the symbols \\
\hline fpc-usage-1 & prop & $\mathrm{f}(\mathrm{z})$ & $\mathrm{u}[\mathrm{f}(\mathrm{z})]$ & Fig.1 & $\begin{array}{l}\text { Calculates propagation of the } \\
\text { uncertainties of the FPC for } \\
\mathrm{f}(\mathrm{z}) \text { according to the law: } \\
u\left[f\left(z_{1}, \ldots, z_{n}\right)\right]= \\
\sqrt{\sum_{i, j=1}^{N} \frac{\partial f}{\partial z_{i}} \operatorname{cov}\left[z_{i}, z_{j}\right] \frac{\partial f}{\partial z_{j}}}\end{array}$ \\
\hline fpc-usage-1 & info & const & $\begin{array}{c}\text { name, unit, } \\
\text { symbTeX }\end{array}$ & Fig.1 & $\begin{array}{l}\text { name — 'const's name; unit } \\
\text { — 'const's unit; symbTeX - } \\
\text { 'const's TeX symbol }\end{array}$ \\
\hline
\end{tabular}

\subsection{Modules with Physical Formulae Collection}

These modules contain some realization of several physical functions used in FPC-1998 evaluations.

\begin{tabular}{|c|c|l|}
\hline Function name & Mathem. symbol & \multicolumn{1}{c|}{ Description } \\
\hline \hline E1tot & $E_{X}(n, \mathrm{~L}, j)$ & Energy levels of hydrogen and deuterium[1] \\
\hline$a_{e}$ & $a_{e}$ & Electron magnetic moment anomaly [1] \\
\hline$a_{\mu}$ & $a_{\mu}$ & Muon magnetic moment anomaly [1] \\
\hline$\Delta \nu_{M u}$ & $\Delta \nu_{M u}$ & Muonium ground-state hyperfine splitting [1] \\
\hline$\nu$ & $\nu$ & $\begin{array}{l}\text { Function conjugated to muonium } \\
\text { ground-state hyperfine splitting([1], p.387) }\end{array}$ \\
\hline
\end{tabular}




\begin{tabular}{|c|c|c|c|l|}
\hline Module & Func. & Input & Example & \multicolumn{1}{|c|}{ Description of the symbols } \\
\hline \hline cod-15-3 & E1tot & $\mathrm{X}, \mathrm{n}, \mathrm{L}, \mathrm{j}$ & Fig. 2 & $\begin{array}{l}\mathrm{X}=(1 \text { or } 2), \mathrm{X}=1 \text { for hydrogen and } \\
\mathrm{X}=2 \text { for deuterium; } \mathrm{n} \text { - principal } \\
\text { quantum number; L - nonrelativistic } \\
\text { orbital angular momentum; } \\
\mathrm{j} \text { - angular momentum quantum } \\
\text { number; }\end{array}$ \\
\hline formulae-2 & $a_{e}$ & $\begin{array}{c}\text { alpha, } \\
\text { dae }\end{array}$ & & $\begin{array}{l}\text { alpha - fine structure constant; } \\
\text { dae - value of theoretical uncertainty } \\
\text { for } a_{e} \text { (see [1], p.476). }\end{array}$ \\
\hline formulae-2 & $a_{\mu}$ & $\begin{array}{c}\text { alpha, } \\
\text { damu }\end{array}$ & & $\begin{array}{l}\text { alpha - fine structure constant; } \\
\text { damu - value of theoretical uncertainty } \\
\text { for } a_{\mu} \text { (see [1], p.479). }\end{array}$ \\
\hline formulae-2 & $\Delta \nu_{M u}$ & $\begin{array}{l}\text { mE, mM, } \\
\text { aMU, } \\
\text { alpha, R }\end{array}$ & Fig.3 & $\begin{array}{l}\text { alpha - fine structure constant; } \\
\text { mE - electron mass; mM - muon } \\
\text { mass; R - Rydberg constant. } \\
\text { aMU - Muon magnetic moment } \\
\text { anomaly }\end{array}$ \\
\hline
\end{tabular}

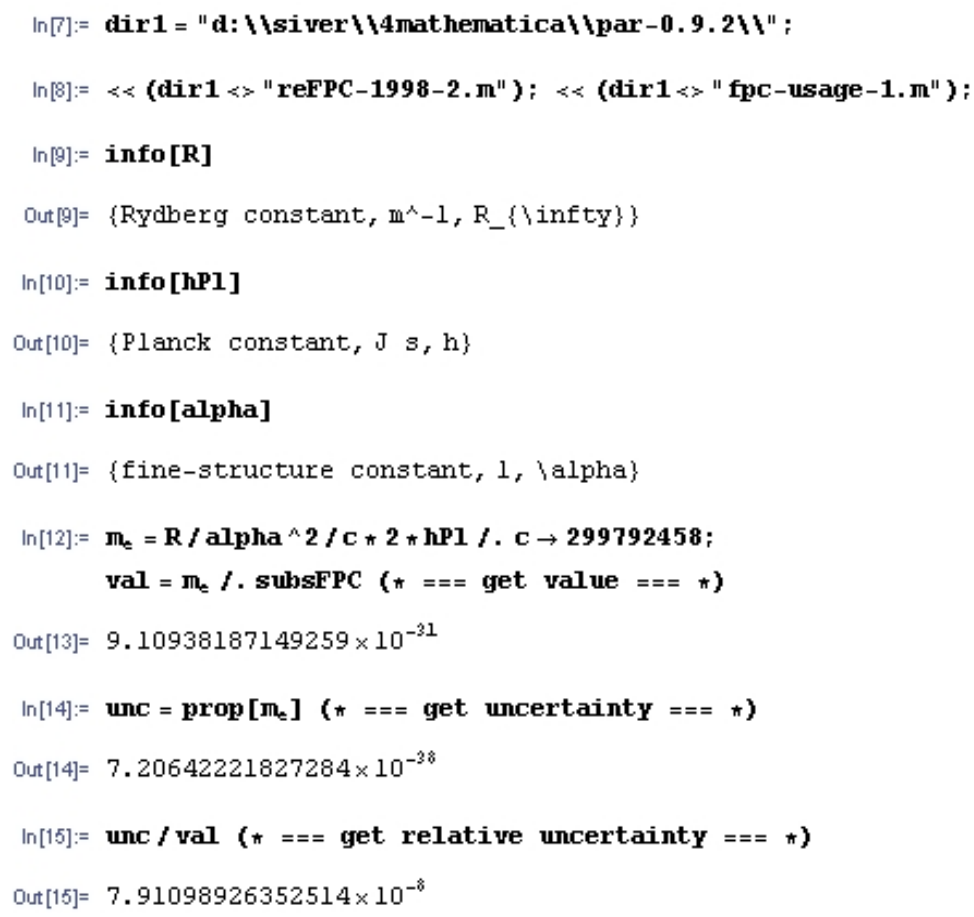

Fig. 1. Example of the usage of modules for calculations with the FPC 


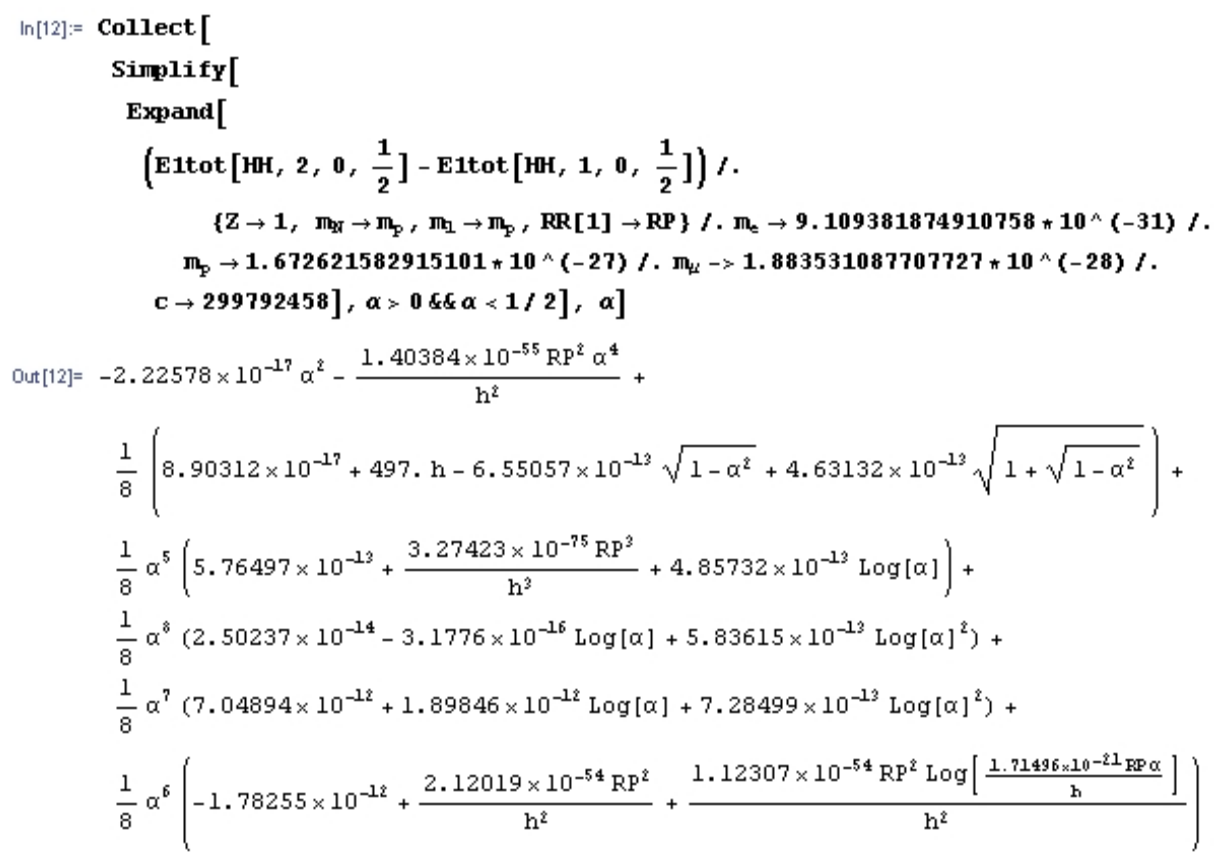

Fig. 2. Lamb shift. Example of the usage of the 'E1tot' function. Unfortunately, it's impossible to show the expression for classical Lamb shift in a reasonable form obtained as Mathematica output. So we make extra substitutions for $\mathrm{m}_{\mathrm{e}}, \mathrm{m}_{\mathrm{p}}, \mathrm{m}_{\mu}$ and $\mathrm{c}$ using the values of FPC-1998.

\section{Further Work. Discussion}

A long time ago it was realized that evaluation of the FPC gave two important results: (i) values of the FPC and (ii) test for modern theories on agreement with each other and with experiments [1].

The first task can be solved with the help of PAREVAL package. But in order to solve the second one a system for monitoring the values of FPC should be created. This system should include:

- Collection of methods for adjustment of parameters of the theories;

- A database of all measured experimental data which can be used in parameters evaluation;

- A database of all self-consistent modern theoretical formulae, relevant to the experimental database. Besides, the database of the formulae should be connected to calculation media;

- Collection of methods that could test statistical hypotheses and seek possible systematical errors or uncertainties of calculation methods and programming "bugs";

- Subsystem for the presentation of results; 


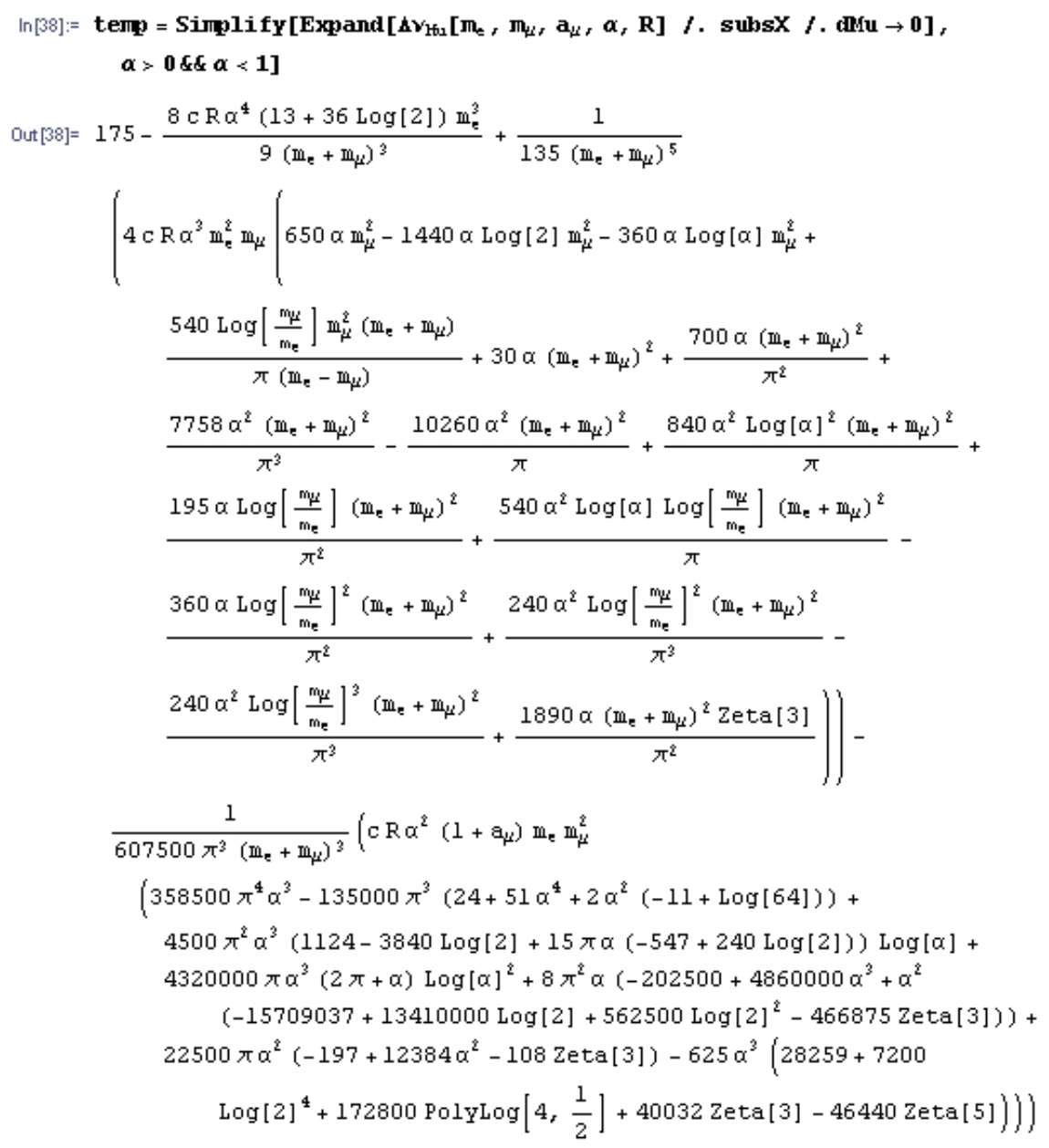

Fig. 3. Example of the usage of the ' $\Delta \nu_{M u}$ ' function - muonium ground-state hyperfine splitting

- Subsystem for automatical or semi-automatical search for scientific information (old, modern and which just have appeared), which can be helpful to researcher.

As far as we know, none of such systems actually exists. PAREVAL package can be considered as a prototype of such system.

At last we would like to note that our working experience in Mathematica tells us that this computer algebra system is powerful enough to realize a system for monitoring values of fundamental physical constants. 


\section{References}

1. P. J. Mohr and B. N. Taylor, "CODATA recommended values of the fundamental physical constants: 1998", Rev. Mod. Phys. 72 (2000) 351.

2. Mathematica, http://www.wolfram.com

3. Fundamental Physical Constants from NIST, http://physics.nist.gov/cuu/Constants/

4. Siver A.S., Ezhela V.V., "On the CODATA recommended values of the fundamental physical constants: V3.2(1998)\&V4.0(2002)", IHEP Preprint 2003-34, Protvino, 2003; arXiv:physics/0401064

5. For General Public License (GPL) note see http://www.gnu.org 